\title{
A Sliding law for Glaciers of Constant Viscosity in the Presence of Subglacial Cavitation
}

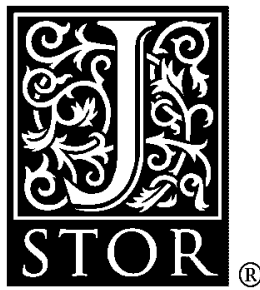

\author{
A. C. Fowler \\ Proceedings of the Royal Society of London. Series A, Mathematical and Physical Sciences, Vol. \\ 407, No. 1832, Predictability in Science and Society. (Sep. 8, 1986), pp. 147-170.
}

Stable URL:

http://links.jstor.org/sici?sici=0080-4630\%2819860908\%29407\%3A1832\%3C147\%3AASLFGO\%3E2.0.CO\%3B2-8

Proceedings of the Royal Society of London. Series A, Mathematical and Physical Sciences is currently published by The Royal Society.

Your use of the JSTOR archive indicates your acceptance of JSTOR's Terms and Conditions of Use, available at http://www.jstor.org/about/terms.html. JSTOR's Terms and Conditions of Use provides, in part, that unless you have obtained prior permission, you may not download an entire issue of a journal or multiple copies of articles, and you may use content in the JSTOR archive only for your personal, non-commercial use.

Please contact the publisher regarding any further use of this work. Publisher contact information may be obtained at http://www.jstor.org/journals/rsl.html.

Each copy of any part of a JSTOR transmission must contain the same copyright notice that appears on the screen or printed page of such transmission.

JSTOR is an independent not-for-profit organization dedicated to and preserving a digital archive of scholarly journals. For more information regarding JSTOR, please contact support@jstor.org. 


\title{
A sliding law for glaciers of constant viscosity in the presence of subglacial cavitation
}

\author{
BY A. C. FowleR \\ Mathematical Institute, 24-29 St Giles', Oxford OX1 3LB, U.K. \\ (Communicated by J. F. Nye, F.R.S. - Received 10 October 1985 - \\ Revised 20 March 1986)
}

\begin{abstract}
A method of solution for the problem of slow flow of a Newtonian viscous glacier slipping over a rough bed is constructed, for the case where cavities form when the lubricating water film pressure reaches that of the local subglacial drainage system. The treatment of Nye (Proc. R. Soc. Lond. A 311, 445-477 (I 969)) is reformulated as a Hilbert problem, and the solution presented for the particular case of a periodic bedrock with one cavity per period. For such bedrocks, it is found that the basal stress has a maximum for a finite basal velocity, and the basal stress decreases towards zero as the velocity tends to infinity, in line with the suggestion of Lliboutry (J. Glaciol. 23, 67-95 (r 979)). For more complicated bedrocks, with many different obstacle sizes, direct solution appears impractical and some kind of further approximation seems advisable.
\end{abstract}

\section{INTRODUCTION}

Near the base of an actively flowing temperate glacier (i.e. one whose ice is at the pressure-melting point), neither the velocity nor the shear stress tend to zero, which would be the case for either in more familiar fluid mechanical situations. Instead, the ice flows because of a thin, lubricating film of water, which exerts zero stress locally at the base. However, the traction a few metres above the bedrock is non-zero because of the resistance to the flow offered by the roughness of the bedrock caused by varying normal pressures there.

To formulate an appropriate basal boundary condition for the larger scale ice flow, it is necessary to study the local flow near the bedrock. By matching the solution for this local flow to the larger scale ice flow, one obtains a boundary condition for the latter flow, called the sliding law:

$$
\tau_{\mathrm{b}}=f\left(u_{\mathrm{b}}\right)
$$

were $\tau_{\mathrm{b}}$ is the effective basal shear stress, and $u_{\mathrm{b}}$ the effective basal velocity.

The determination of the form of this function has engendered a substantial literature (Weertman 1957, I964, I97 I, 1979; Lliboutry 1968, 1975, 1976, 1978, 1979; Morland 1976a, $b$; Kamb 1970; Nye 1969, I970; Fowler 1981 ; Iken 198 I), but the difficulty of the problem has ensured a lack of conclusiveness in the results. Nevertheless, there is much continuing interest in determining the sliding law, because of the importance its form may have in the theoretical study of surges and seasonal waves (Hutter r $982 a$; Fowler r 982). 
Let us summarize the basic physics involved, to put the problem here in its context. Ice flows slowly (i.e. zero Reynolds number) over a rough bedrock $h(x)$. Already we have implicitly restricted our discussion to the case of an undeformable bed, whereas many glaciers probably flow over beds of deformable sediments, caused by constant erosion by the ice. The particular problem addressed here is nevertheless worthy of study. In the basal layer, the flow is driven by an applied velocity 'at infinity', and experiences zero traction at the rough interface. There is a normal velocity at the interface because of melting there, which is due to a back flux of temperature in the bedrock owing to the pressure variation in the ice (that affects the melting temperature). Since the vertical velocity 'at infinity' is prescribed (zero), the stress 'at infinity' can (in principle) be calculated, as in (1.1).

The main complications involved in solving the above problems are the following:

(i) Nonlinear flow law. The simplest realistic rheological flow law for ice is Glen's law, which says that the viscosity $\eta$ varies with a power of the second stress invariant $\tau^{2}=\frac{1}{2} \tau_{i j} \tau_{i j}, \tau_{i j}$ being the stress deviator tensor. Often, one takes

$$
\eta=1 / A \tau^{n-1},
$$

with $n \approx 3$. This renders the ice-flow problem fairly intractable by analytical methods, although it is possible to find variational principles, which can be used in an approximate manner (Fowler I98I; Oakberg r 98I).

(ii) Finite bedrock slope. Obviously, a solution will be easier in a half space $y>0$, than in a 'corrugated' half space $y>h(x)$; thus an analysis which assumed 'small' bedrock slopes is attractive. This was the basis of the solutions given by Nye (r 969 , 1970). It was pointed out by Fowler (1981), that the proper formulation of the sliding law as a boundary condition requires the assumption $[x] \ll d$, where $[x]$ is the 'wavelength' of the micro-relief $(h)$, and $d$ is the glacier depth, and that in this case the basal velocity $u_{\mathrm{b}}$ will only (asymptotically) be significant if the micro-aspect ratio $(c a .|\mathrm{d} h / \mathrm{d} x|)$ is asymptotically of order $\sigma^{1 /(n+1)}$, where

$$
\sigma=[x] / d
$$

and $n$ is the constant in Glen's flow law. In other words, it is self-consistent, from the point of view of formulation, to have either $u_{\mathrm{b}} \approx 0$, or determine $f\left(u_{\mathrm{b}}\right)$ by using a small-slope analysis.

(iii) Regelation. In solving the ice-flow problem, we have alluded to a boundary condition for the ice velocity at the ice-rock interface, of the form

$$
v_{\mathrm{n}}=-\delta_{\mathrm{r}} V_{\mathrm{m}}(x),
$$

where $v_{\mathrm{n}}$ is the normal component of the ice velocity at the interface and $V_{\mathrm{m}}$ is a melting velocity determined by the heat flux at the interface; if $v_{\mathrm{n}}$ and $V_{\mathrm{m}}$ are scaled to be $O(1), \delta_{\mathrm{r}}$ is a measure of the importance of this regelative velocity to the ice-flow. A fairly conservative estimate of $\delta_{\mathrm{r}}$, based on microslopes ca. 0.2 and a sliding velocity $c a .10 \mathrm{~m} \mathrm{a}^{-1}$, is

$$
\delta_{\mathrm{r}} \approx 10^{-3} /[x],
$$


where $[x]$ is measured in metres (Fowler $\mathrm{I} 98 \mathrm{I}$ ). We see that typically $\delta_{\mathrm{r}}$ is negligible, if $[x] \sim 1 \mathrm{~m}$, for example. Neglect of $\delta_{\mathrm{r}}$ in (1.4) uncouples the ice-flow problem (which renders the solution more tractable), and is equivalent to the assumption that very small scale roughness is absent; however, if such roughness is present, then the regelative component of the flow is important. In what follows, we formally suppose that small scale roughness is absent, and therefore ignore regelation altogether, primarily for clarity of exposition.

(iv) Films, layers and cavities. The water film is the lubrication film (thickness $\sim 10^{-6} \mathrm{~m}$ ) that is considered to separate ice and bedrock. By using lubrication theory, one can show that the (scaled) film thickness $\Sigma(x)$ satisfies

$$
\Sigma^{3}=-12\left\{\int_{x_{0}}^{x} V_{m}\left(x^{\prime}\right) \mathrm{d} x^{\prime}\right\} / p^{\prime}(x),
$$

(Fowler $198 \mathrm{I}$ ) for the case of small bedrock slope, where $V_{\mathrm{m}}$ is as above and $p$ is the water pressure, which may be taken equal to the ice pressure (see later analysis) in the present instance. Since $\Sigma$ uncouples from the ice-rock problem, it would be of no further interest, except that $\Sigma \rightarrow 0$ as $\int_{x_{0}}^{x} V_{\mathrm{m}} \rightarrow 0, \Sigma \rightarrow \infty$ as $p^{\prime} \rightarrow 0$. In the first case, the film becomes pinched out, and one must have a cold patch (Robin I 976) in which, presumably, the no-slip condition (or a Coulomb friction law) applied. In the second case (analogous to boundary layer separation in high Reynolds number flows!), the ice-water interface leaves the proximity of the bedrock, and one has a cavity with an (unknown) free boundary, which is determined by the extra condition $p=$ constant in the cavity. We will term this phenomenon film separation and call the 'cavity' a separated layer, to distinguish it from the more usual type of cavity discussed below. A similar kind of behaviour arose in Nye (1973), and is discussed by Fowler (198I). The pressure in such separated layers is determined by the film dynamics. Therefore, if these layers have access to the subglacial drainage system, a further complicated interaction will occur. Such layers might (if they exist) provide a means of drowning small scale roughness, although Weertman's (1972, 1979) water layer for this purpose was a sheet-like constituent of the drainage system itself.

The term 'cavity' is usually reserved for separation of the ice-rock interface due to the water (or ice) pressure becoming too low. In an extreme case, where there is no connection to the subglacial drainage system, this would be when the water pressure reaches the triple point. In the more realistic case, where the interface has access to the drainage system, the criterion for separation would be that the water pressure reaches the local drainage pressure, as determined (for example) by Röthlisberger's ( 1972) theory. The cavity has a free (unknown) boundary, and the extra constant pressure condition (in principle) determines this.

(v) Other physical mechanisms. Further complications include possible generalizations of the rheological flow law (e.g. transient creep, dependence of viscosity on water content, incorporation of basil till/deformable sediments). An important effect is that of moisture. Since it is temperate ice which slides, and since temperate ice is moist ice (Lliboutry 1976), the transport of moisture within basal ice can be expected to affect the amount of water at the bed, and consequently the sliding velocity; it is indeed well known from field observations that this is the case (see, 
for example, (Hodge 1974)). This is because the supply of water to the bed (drainage) may affect the basal water pressure (via Röthlisberger-type analysis) and hence the effective (overburden minus water) pressure, which is a controlling parameter in the sliding law in the presence of cavitation (Lliboutry 1979). However, the theory of moisture transport in temperate ice is still in a fairly rudimentary state (Lliboutry 1976, Hutter 1982 $b$, Fowler 1984).

In this paper, we make an attack on the form of the sliding law in the presence of cavitation. We will present an extension of the Nye-Kamb theory to the case where cavities can form, as discussed above (Weertman I 979, pp. 106, 122). That is, we ignore complications mentioned in (v) above, and assume $(a)$ a linear flow law (ignore (i)); (b) small bedrock slope (ignore (ii), but with good reason); (c) neglect regelation, or equivalently, small scale roughness (ignore (iii)), although the method presented here should also be able to deal (in principle) with that case; and $(d)$ include cavitation but ignore film separation. With these assumptions the problem as stated can be solved, in the sense that it can be reduced to a quite manageable numerical problem, which is essentially of algebraic type. It is nonlinear, because of the free-boundary nature of the formulation, and consequently the most general case cannot be usefully treated.

\section{Reformulation of the Nye-Kamb theory as a HILBERT PROBLEM}

We consider the slow flow of an incompressible Newtonian fluid over a 'slowly-varying' rough bedrock, inclined at a mean angle $\theta$ to the horizontal, as shown in figure 1. By slowly-varying and rough, we explicitly mean that the equation of the bedrock can be written in the form

$$
y=d h_{\mathrm{s}}(x / l)+\nu[x] h(x /[x]),
$$

where $d$ is a measure of the typical glacier thickness, $l$ is a typical length over which $h_{\mathrm{s}}$ varies by $O(1)$, and similarly, $\nu[x]$ measures the amplitude of the rough bed, and $[x]$ is its 'wavelength'. The nature of the bed is then essentially specified by the two parameters $v$ and

$$
\sigma=[x] / d ;
$$

$\nu$ measures the roughness of the bed, whereas $\sigma$ is a measure of how 'grainy' or corrugated the bed appears on the large scale. We explicitly assume that $\sigma \ll 1$. The flow is assumed two-dimensional.

If the velocity components are $(u, v)$, we can introduce a stream function $\psi$ by

$$
u=\psi_{y}, \quad v=-\psi_{x},
$$

where here and following, $x$ and $y$ subscripts denote partial derivatives. It is convenient to non-dimensionalize the variables in the following way (Fowler 198I):

$$
\left.\begin{array}{rl}
x & =[x] x^{*}, \quad y=h_{\mathrm{s}}+[x] y^{*}, \\
p & =p_{\mathrm{A}}+\rho g(Y-y) \cos \theta+[\tau] p^{*} / \nu, \\
\psi & =U[x]\left(u_{\mathrm{b}} y^{*}+\nu \psi^{*}\right),
\end{array}\right\}
$$




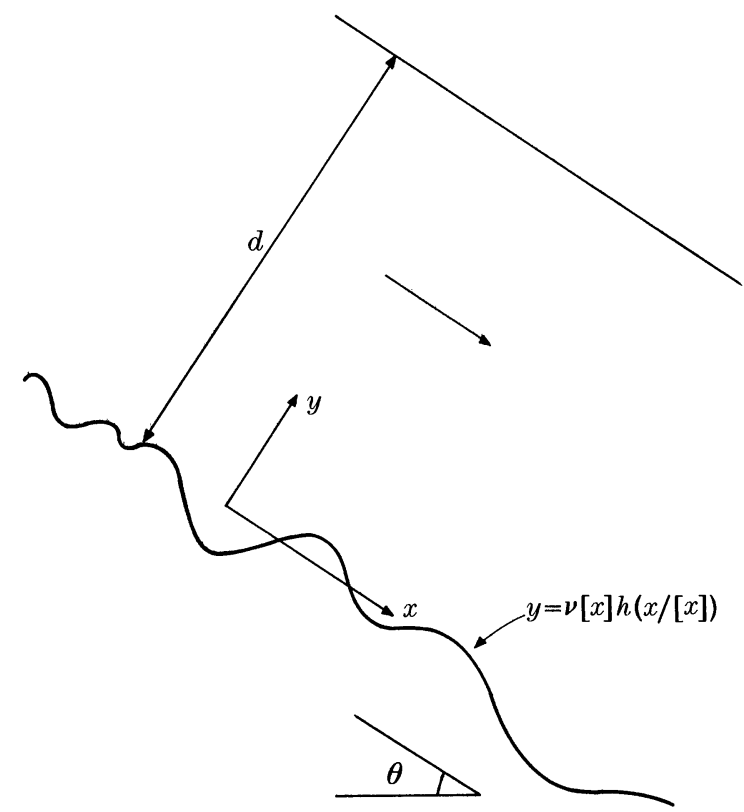

Figure 1. Flow geometry. On the scale of the figure, $h_{\mathrm{s}}$ is essentially constant.

where $Y$ is the top surface, $p_{\mathrm{A}}$ is atmospheric pressure, $\rho$ is density, $g$ is gravity and $[\tau]$ and $U$ are stress and velocity scales given by

$$
[\tau]=\rho g d \sin \theta=\eta U / d,
$$

$\eta$ being the ice viscosity, which we assume is constant. Furthermore we may define

$$
\nu=\sigma^{\frac{1}{2}}
$$

which leads to a correct scaling of the problem (Fowler 198I), and is consistent with results of Richardson (1973). Then appropriate equations governing the basal flow are, omitting the asterisks on the dimensionless variables for convenience,

$$
p_{x}=\nu^{3}+\nabla^{2} \psi_{y}, \quad p_{y}=-\nabla^{2} \psi_{x}
$$

Boundary conditions 'at infinity' are found by matching to the outer flow in $y \sim 1 / \sigma$. If the dimensionless 'basal' stress perceived by the outer flow is $\tau_{\mathrm{b}}$ (i.e. in units of $[\tau]$ ), then $\psi$ satisfies (Fowler. 198I)

$$
\psi \sim \frac{1}{2} \nu \tau_{\mathrm{b}} y^{2}, \quad y \rightarrow \infty .
$$

At the bedrock, we prescribe zero shear stress and no flow through (neglect regelation). These conditions can be written (Fowler 198I)

$$
\left.\begin{array}{c}
\psi=-u_{\mathrm{b}} h(x), \\
\left(1-\nu^{2} h^{\prime 2}\right)\left(\psi_{y y}-\psi_{x x}\right)-4 \nu h^{\prime} \psi_{x y}=0,
\end{array}\right\}
$$


on $y=\nu h$. Equations (2.7) to (2.9) constitute the problem to be solved. At leading order in $\nu$, this is the following:

$$
\left.\begin{array}{c}
p_{x}=\nabla^{2} \psi_{y}, \quad p_{y}=-\nabla^{2} \psi_{x} ; \\
\psi \sim 0, \quad y \rightarrow \infty ; \quad \psi=-u_{\mathrm{b}} h(x), \quad \psi_{y y}-\psi_{x x}=0, \quad \text { on } \quad y=0 .
\end{array}\right\}
$$

(More generally, $\psi \sim$ const. at infinity, but this can be taken to be zero if the origin of $y$ is chosen appropriately.) Notice that $\psi$ is determined in terms of $u_{\mathrm{b}}$, but $\tau_{\mathrm{b}}$ does not appear at this order. Nevertheless, it can be determined from the solution to (2.10); by taking a force balance (integrate (2.7) over $y>\nu h$ ), assuming that longitudinal stresses balance (which can be guaranteed by taking $h$ periodic, and specifying periodicity of $\psi$ and its first, second and third derivatives (Fowler 198I) one finds, from (2.8) and the divergence theorem (on the integral of (2.7)), that

$$
\tau_{\mathrm{b}}=\left.(1 / 2 \pi) \int_{0}^{2 \pi}\left[p+2 \psi_{x y}\right]\right|_{y=0} h^{\prime} \mathrm{d} x
$$

where henceforth we will assume periodicity of $h$ with a period $2 \pi$. Equation (2.11) is no more than the intuitively obvious balance of stress at infinity by normal stress variation at the bedrock. In (2.11), $p+2 \psi_{x y}$ is just $-\sigma_{n n}$ where $\sigma_{n n}$ is the (scaled) compressive stress. Consequently, continuity of the stress tensor at the ice-water interface yields

$$
\left.\left(p+2 \psi_{x y}\right)\right|_{y=0}=p_{\mathrm{w}}(\text { scaled }),
$$

where $p_{\mathrm{w}}$ is the dimensionless water pressure.

It will be seen that the formulation of the problem will naturally lead to a description of $\tau_{\mathrm{b}}$ as a function of $u_{\mathrm{b}}$, as in (1.1).

So far we have not mentioned the possibility of cavitation. Cavities will form if the water film (dimensional) pressure decreases to $p_{\mathrm{h}}$, the local subglacial hydrological drainage pressure. If this happens, $h(x)$ is taken along the cavity roof, and is unknown there; however, there is an extra condition, that the cavity pressure equals the drainage value, $p_{\mathrm{h}}$, which is a dimensional quantity. From (2.4), this condition is written in terms of the dimensionless pressure $p$ as

$$
p_{\mathrm{A}}+\rho g^{\prime} d[H-\sigma \nu h]+[\tau] p / \nu=p_{\mathrm{h}},
$$

where $H$ is the dimensionless (with $d$ ) depth, and $g^{\prime}=g \cos \theta$. We will return to this in due course. For the moment, we leave aside the problem of cavitation, and turn to the formulation of (2.10) and (2.11) in terms of complex variables.

Elimination of $p$ from the equations shows that $\psi$ satisfies the equation

with solution

$$
\nabla^{4} \psi=0
$$

$$
\psi=\left(z^{*}-z\right) f(z)-B(z)+\left(z-z^{*}\right) f^{*}\left(z^{*}\right)-B^{*}\left(z^{*}\right),
$$

where $f(z)$ and $B(z)$ are arbitrary analytic functions of $z=x+\mathrm{i} y$, and $*$ denotes the complex conjugate. The zero stress condition of $z=z^{*}=x$ requires

$$
B^{\prime \prime}+2 f^{\prime}+B^{* \prime \prime}+2 f^{\prime \prime}=0
$$


which we can satisfy by choosing

Then we require

$$
f(z)=-\frac{1}{2} B^{\prime}(z)
$$

$$
B \rightarrow 0 \quad \text { as } \quad \operatorname{Im} z \rightarrow+\infty
$$

(since $\psi \rightarrow 0$ at $\infty$ ) and the condition $\psi=-u_{\mathrm{b}} h$ on $y$ implies

$$
B+B^{*}=u_{\mathrm{b}} h \quad \text { on } \quad \operatorname{Im} z=0 .
$$

Thus the problem is reduced (for given $h$ ) to finding an analytic function $B(z)$ in $\operatorname{Im} z>0$ satisfying (2.18) and (2.19).

Notice that if $h$ is piecewise continuous, then it has a Fourier series

$$
h=\sum_{-\infty}^{\infty} a_{k} \mathrm{e}^{\mathrm{i} k x}
$$

with $a_{0}=0$ (this chooses the origin of $y$ so that the mean value of $h$ is zero). By inspection, the function $B$ is then given by

$$
B(z)=u_{\mathrm{b}} \sum_{1}^{\infty} a_{k} \mathrm{e}^{\mathrm{i} k z}
$$

When cavities are present, the coefficients $a_{k}$ are not known (since $h$ is not completely known), but the representations (2.20) and (2.21) will still be valid.

We can formulate a Hilbert problem as follows. First, note that $B^{\prime \prime}$ satisfies

$$
B^{\prime \prime}+B^{* \prime \prime}=u_{\mathrm{b}} h^{\prime \prime} \quad \text { on } \quad \operatorname{Im} z=0 .
$$

From (2.15), and using (2.17), we find $\nabla^{2} \psi=4 \partial^{2} \psi / \partial z \partial z^{*}=-2\left(B^{\prime \prime}+B^{* \prime \prime}\right)$; also since $(2.10)_{1,2}$ are the Cauchy-Riemann equations for $p+\mathrm{i} \nabla^{2} \psi$, it follows that this last expression is analytic, say $S(z)$, so that $p=S(z)-\mathrm{i} \nabla^{2} \psi=S(z)-2 \mathrm{i}\left(B^{\prime \prime}+B^{* \prime \prime}\right)$. But $p$ is real, and it immediately follows that in fact

$$
p=-2 \mathrm{i}\left(B^{\prime \prime}-B^{* \prime \prime}\right),
$$

plus an analytic function, which (since it is real) must be a constant, and can be taken as zero by matching $p$ to the outer solution.

Now define

$$
\left.\begin{array}{l}
L(z)=B^{\prime \prime}(z), \quad \operatorname{Im} z>0, \\
L(z)=\left[B^{\prime \prime}\left(z^{*}\right)\right]^{*}, \quad \operatorname{Im} z<0,
\end{array}\right\}
$$

where $L$ is an analytic function in $\operatorname{Im} z>0$ and $\operatorname{Im} z<0$. Denote by

$$
\left.\begin{array}{l}
L^{+}(x)=\lim _{\operatorname{Im} z \rightarrow 0+} L(z), \\
L^{-}(x)=\lim _{\operatorname{Im} z \rightarrow 0-} L(z),
\end{array}\right\}
$$

then $(2.22)$ is

$$
L^{+}+L^{-}=u_{\mathrm{b}} h^{\prime \prime}
$$

and $(2.23)$ is

$$
L^{+}-L^{-}=\frac{1}{2} \mathrm{i} p \text {. }
$$


If $h$ is known (no cavities), (2.26) is a Hilbert problem for the function $L(z)$, together with conditions

$$
L \rightarrow 0, \quad \operatorname{Im} z \rightarrow \pm \infty .
$$

Since $h$ is periodic, it is useful to transform from the $z$ plane to the $\zeta$ plane, $\zeta=\mathrm{e}^{\mathrm{i} z}$. We define

also

$$
\begin{gathered}
\xi=\mathrm{e}^{\mathrm{i} x}, \\
h(x) \equiv \tilde{h}(\xi), \\
h^{\prime}(x) \equiv h_{1}(\xi), \\
h^{\prime \prime}(x) \equiv h_{2}(\xi)=-\xi\left(\xi h_{1}\right)^{\prime} ; \\
\zeta=\mathrm{e}^{\mathrm{i} z}, \quad F(\zeta) \equiv L(z) ;
\end{gathered}
$$

denote $D^{+}$the interior $|\zeta|<1$ of the unit circle $C_{1}, D^{-}$is the exterior $|\zeta|>1$. Then we have

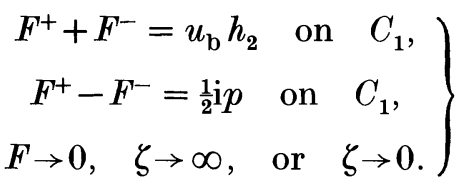

Further, it follows from (2.15) and (2.17) that $\psi_{x y}=0$ on $y=0$; consequently $\left.p\right|_{y=0}=p_{\mathrm{w}}$, and the drag law (2.11) is

$$
\left.\begin{array}{rl}
\tau_{\mathrm{b}} & =\left.(1 / 2 \pi) \int_{0}^{2 \pi} p\right|_{y=0} h^{\prime} \mathrm{d} x \\
& =(1 / \mathrm{i} \pi) \int_{0}^{2 \pi}\left(L^{+}-L^{-}\right) h^{\prime} \mathrm{d} x \\
& =(1 / \mathrm{i} \pi) \int_{C_{1}}\left(F^{+}-F^{-}\right) h_{1}(\xi) \mathrm{d} \xi .
\end{array}\right\}
$$

For the case of no cavitation, when $h$ is given by $(2.20)$, we define

$$
\begin{gathered}
D(z)=\sum_{1}^{\infty} a_{k} \mathrm{e}^{\mathrm{i} k z}, \quad a_{-k}=a_{k}^{*}, \\
B=u_{\mathrm{b}} D, \\
\left.p\right|_{0}=-2 \mathrm{i} u_{\mathrm{b}}\left[D^{\prime \prime}-D^{* \prime \prime}\right], \\
h^{\prime}=D^{\prime}+D^{*^{\prime}},
\end{gathered}
$$

and hence, after a little reduction,

or

$$
\begin{gathered}
\tau_{\mathrm{b}}=2 u_{\mathrm{b}} / \mathrm{i} \pi \int_{0}^{2 \pi} D^{*^{\prime}} D^{\prime \prime} \mathrm{d} x, \\
\tau_{\mathrm{b}}=4 u_{\mathrm{b}} \sum_{1}^{\infty} k^{3}\left|a_{k}\right|^{2},
\end{gathered}
$$

which is the non-cavitational formula, when regelation is ignored (Nye 1969).

Now suppose that a part $C$ of the bedrock is cavitated: Denote the cavity-free part by $C^{\prime}$. Then in $C, h$ is unknown, but the pressure is given by

$$
p=-p_{c} \text { in } C \text {, }
$$


where from (2.5) and (2.13), it follows (neglecting $O(\sigma \nu))$ that

$$
p_{c}=\frac{\nu}{\tan \theta}\left[\frac{P-p_{\mathrm{h}}}{\rho g^{\prime} d}\right] \text {. }
$$

Here $P$ is the overburden pressure $p_{\mathrm{A}}+\rho g^{\prime} d Y=P$, and thus $P-p_{\mathrm{h}}=N$, the effective pressure at the bed. Its introduction into the sliding law in the presence of cavitation is due to Lliboutry (e.g. 1968). Notice that the dependence of the sliding law on $p$, and hence $N$ is derived here, and not simply assumed. The dimensionless underpressure $p_{c}$ is reasonably $O(1)$, for reasonable bedrock roughness and mean slope. Now the formulation above is still valid; however, since $h^{\prime \prime}$ is not known in $C$, but $p$ is, we have a mixed Hilbert problem of the following form (see $(2.31))$ :

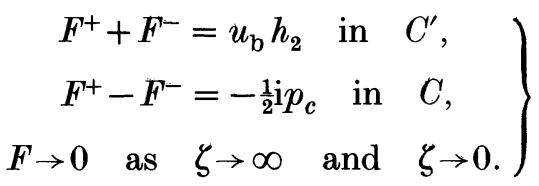

Once the solution of (2.41) is obtained, the drag can be obtained from (2.32).

Mixed problems of the above type are common in linear elasticity (England r97 I), the only difference here being that the location of $C$ is not known; to determine this, the cavity roof shape must be solved for, as well as (2.48). This added complexity makes the problem slightly different to the standard mixed problem, and so we describe the method of solution used here in the following section.

\section{Method OF SOLUTION}

To facilitate the solution of (2.41), we define

$$
\left.\begin{array}{ll}
G(\zeta)=F(\zeta)+\mathrm{i} p_{c} / 4, & \zeta \text { in } D^{+} \\
G(\zeta)=F(\zeta)-\mathrm{i} p_{c} / 4, & \zeta \text { in } D^{-}
\end{array}\right\}
$$

then

$$
\left.\begin{array}{rl}
G^{+}+G^{-} & =u_{\mathrm{b}} h_{2} \quad \text { on } \quad C^{\prime} \\
G^{+}-G^{-} & =0 \quad \text { on } \quad C, \\
G(\infty) & =-\mathrm{i} p_{c} / 4, \quad G(0)=\mathrm{i} p_{c} / 4 .
\end{array}\right\}
$$

Thus $G$ is analytic except on $C^{\prime}$, which is thus a branch cut for $G$ (see figure 2). For given $C^{\prime},(3.2)$ is a standard Hilbert problem. Note that

$$
\left.\begin{array}{rl}
G^{+}+G^{-} & =u_{\mathrm{b}} h_{2}^{C}, \\
p+p_{c} & =(2 / \mathrm{i})\left(G^{+}-G^{-}\right) \text {in } C^{\prime}, \\
\tau_{\mathrm{b}} & =(1 / \mathrm{i} \pi) \int_{C^{\prime}}\left(G^{+}-G^{-}\right) h_{1} \mathrm{~d} \xi .
\end{array}\right\}
$$

Here $h_{2}^{C}$ points out the fact that $h_{2}=h^{\prime \prime}$ is taken along the cavity roof. 


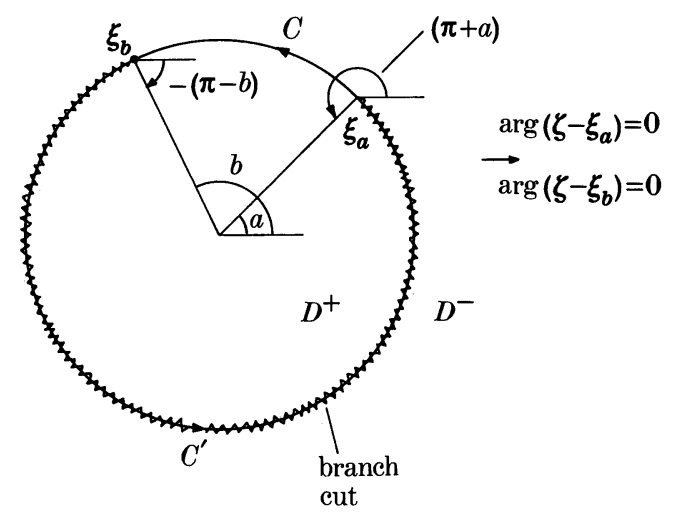

Figure 2. Contours of $C$ and $C^{\prime}$ in the complex $\zeta$ space. There is a branch cut for $\chi$ along $C^{\prime}$.

The solution of (3.2) (for given $C^{\prime}$ ) is unique, and can be written in various ways (England I97 I). By inspection, one such way is

$$
G=\frac{1}{2} u_{\mathrm{b}}\left[h_{2}-q \chi\right],
$$

where

$$
\chi^{+}+\chi^{-}=0 \text { on } C^{\prime} \text {, }
$$

(but is analytic otherwise). ( $h_{2}$ is continued to an analytic function in the obvious way.) The function $q(\zeta)$ is chosen in order that $G$ is finite at $\infty$, and $G(\infty)=-\mathrm{i} p_{c} / 4$ (and also so that $G(0)=\mathrm{i} p_{c} / 4$ ). It is analytic, except at 0 and $\infty$, where also $h_{2}$ is singular. It follows from (3.4) and (3.3) that

$$
\left.\begin{array}{ccc}
\left(p+p_{c}\right) / 2 u_{\mathrm{b}}=\mathrm{i} q \chi^{+} & \text {on } & C^{\prime}, \\
H^{\prime \prime}=h_{2}^{C}-h_{2}=-q \chi & \text { on } & C,
\end{array}\right\}
$$

where $H(x)$ is the cavity thickness $\left(h_{2}\right.$ is derived from the given bedrock shape $h$, which is of course known in $C$, whereas $h_{2}^{C}$ is derived from the cavity roof shape).

Before choosing $q$, we must decide on the function $\chi$. The precise choice depends on what singularities represent physically realistic conditions, and is of crucial importance. Let us suppose there is only one cavity per period, $C=(a, b)$. Then the differential equation $(3.6)_{2}$ for $H$ must satisfy two obvious boundary conditions,

$$
H(a)=H(b)=0 .
$$

Further conditions are that $p+p_{c}$ is real, so that i $q \chi^{+}$is real on $C^{\prime}$ and also that $H^{\prime \prime}$ is real, so $q \chi$ is real on $C$; however, we shall show that these two statements are equivalent. There remains one extra condition to be specified, to determine both $a$ and $b$. If $H^{\prime} \neq 0$ at $a$ or $b$, that is the cavity roof slope is not equal to the bedrock slope at either end point, then one might physically expect a singularity for $p+p_{c}$ there. (Actually, one would expect a non-integrable one, of the form $p+p_{c} \sim O(1 /(x-b))$, for example, which is, however, inconsistent with the solution of (3.2).) This suggests at least that we prescribe

$$
H^{\prime}(a)=0,
$$


and that also $p+p_{c}$ is continuous at the upstream end, which seems the physically appropriate place to avoid singularity. Since $p+p_{c}$ has the same singularities as $\chi$, it follows that the corresponding choice of $\chi$ satisfying (3.5) is

$$
\chi=\left(\frac{\zeta-\xi_{a}}{\zeta-\xi_{b}}\right)^{\frac{1}{2}}, \quad \chi \sim 1 \quad \text { as } \quad x \rightarrow \infty,
$$

where

$$
\xi_{a}=\mathrm{e}^{\mathrm{i} a}, \quad \xi_{b}=\mathrm{e}^{\mathrm{i} b}, \quad 0<b-a<2 \pi .
$$

Then we will have $p+p_{c} \sim(a-x)^{\frac{1}{2}}$ near $x=a, p+p_{c} \sim(x-b)^{-\frac{1}{2}}$ near $x=b$. We shall see below that (3.8) and (3.9) lead to a well-posed problem to determine $a$, $b$, and $H(x)$. Other choices of $\chi$, notably $\left(\zeta-\xi_{a}\right)^{\frac{1}{2}}\left(\zeta-\xi_{b}\right)^{\frac{1}{2}}$, are apparently possible, and we shall discuss this further below.

Now let us suppose

$$
h=\sum a_{n} \xi^{n}
$$

is the given bedrock (summation here, as below, is from $-\infty$ to $+\infty$ ). Then

$$
h_{2}=\sum d_{n} \xi^{n}, \quad d_{n}=-n^{2} a_{n} ;
$$

define $f_{i}$ by

$$
\left(1-\xi_{a} u\right)^{-\frac{1}{2}}\left(1-\xi_{b} u\right)^{\frac{1}{2}}=f_{0}+f_{1} u+f_{2} u^{2}+\ldots,
$$

then

$$
G=\frac{1}{2} u_{b} \chi\left[\sum d_{m} \zeta^{m} \sum_{r \geqslant 0} f_{r} / \zeta^{r}-q\right] .
$$

The coefficient of $\zeta^{s}$ in the square-bracketed term is

$$
\sum_{r \geqslant 0} d_{r+s} f_{r}-l_{s}, \quad s \geqslant 1,
$$

where

$$
q(\zeta)=\sum l_{i} \zeta^{i}
$$

Now since $\chi \sim 1$ as $\zeta \rightarrow \infty$, the condition that $G(\infty)$ is finite then implies that (3.15) is zero, therefore

$$
l_{s}=\sum_{r \geqslant 0} d_{r+s} f_{r}, \quad s \geqslant 1 .
$$

In a similar way, $(3.2)_{3}$ leads to

$$
l_{0}=\sum_{r \geqslant 0} d_{r} f_{r}+\mathrm{i} p_{c} / 2 u_{\mathrm{b}} .
$$

To compute $l_{-s}, s \geqslant 1$, we use the requirement that $G$ be analytic at zero. To do so, we first compute $\chi$ at $\zeta=0$. Denoting $\xi_{a}^{\frac{1}{2}}=\mathrm{e}^{\mathrm{i} a / 2}, \quad \xi_{b}^{\frac{1}{2}}=\mathrm{e}^{\mathrm{i} b / 2}$, for $0<b-a<2 \pi$, it is straightforward to show that

$$
\chi(0)=\chi_{0}=-\xi_{a}^{\frac{1}{2}} \xi_{b}^{-\frac{1}{2}},
$$

and then

$$
\chi(1 / w)=\chi_{0} \chi^{*}(w),
$$


where $\chi^{*}$ denotes the complex conjugate. Then

$$
\left.\begin{array}{rl}
G & =\frac{1}{2} u_{\mathrm{b}} \chi\left[h_{2} / \chi-q\right], \\
& =\frac{1}{2} u_{\mathrm{b}} \chi\left[h_{2} / \chi_{0} \chi^{*}(1 / \zeta)-q(\zeta)\right], \\
& =\frac{1}{2} u_{\mathrm{b}} \chi\left[\frac{1}{\chi_{0}} \sum d_{m} \zeta^{m} \sum_{r \geqslant 0} f_{r}^{*} \zeta^{r}-q\right],
\end{array}\right\}
$$

from (3.13). Since $\chi(0)$ is finite, the coefficient of negative powers of $\zeta$ in the square bracketed term must vanish, and hence

$$
l_{-s}=\sum_{r \geqslant 0} d_{-(s+r)} f_{r}^{*}, \quad s \geqslant 1 .
$$

It is straightforward to show from (3.14), (3.18) and (3.21) that $G(0)=G(\infty)^{*}$, so that both parts of $(3.2)_{3}$ are satisfied. Finally,

$$
q(\zeta)=\sum_{s \geqslant 1} \sum_{r \geqslant 0}\left[f_{r} d_{r+s} \zeta^{s}+\frac{1}{\chi_{0}} f_{r}^{*} d_{-r-s} \zeta^{-s}\right]+\sum_{r \geqslant 0} d_{r} f_{r}+\mathrm{i} p_{c} / 2 u_{\mathrm{b}} .
$$

We next evaluate $\chi$ on $C$ and $C^{\prime}$. This is straightforward algebra, which we omit; as before, care must be taken with the argument of $\chi$. One finds

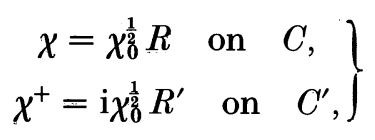

where

where

$$
\left.\begin{array}{rl}
R & =\left[\sin \left(\frac{x-a}{2}\right) / \sin \left(\frac{b-x}{2}\right)\right]^{\frac{1}{2}} \text { on } \quad C, \\
R^{\prime} & =\left[\sin \left(\frac{A-x}{2}\right) / \sin \left(\frac{x-b}{2}\right)\right]^{\frac{1}{2}} \text { on } C^{\prime},
\end{array}\right\}
$$

and

$$
A=a+2 \pi,
$$

Remembering that $d_{-n}=d_{n}^{*}$, it then follows from (3.23), (3.24) and (3.26), that the requirement that $H^{\prime \prime}$ be real on $C$, and that $p$ be real on $C^{\prime}$, are each equivalent to the single condition

$$
\operatorname{Im}\left\{\chi_{0}^{\frac{1}{2}} l_{0}\right\}=0,
$$

where $l_{0}$ is given by (3.18). The cavity thickness is then determined by solving

$$
\left.\begin{array}{c}
H^{\prime \prime}=-R\left[\chi_{0}^{\frac{1}{0}} l_{0}+\sum_{\substack{s \geqslant 1 \\
r \geqslant 0}}\left[f_{r} d_{r+s} \chi_{0}^{\frac{1}{2}} \xi^{s}+(*)\right]\right], \\
H(a)=H^{\prime}(a)=H(b)=0 .
\end{array}\right\}
$$

The stress $\tau_{\mathrm{b}}$ is then computed from $(3.3)_{3}$, which reduces, after some algebra, to the following:

$$
\tau_{\mathrm{b}}=u_{\mathrm{b}} \sum_{n, r} s_{n} m_{r} j_{n+r}
$$


where

$$
\left.\begin{array}{c}
s_{n}=\mathrm{i} n a_{n}, \quad m_{s}=\chi_{0}^{\frac{1}{2}} l_{s}, \quad s \geqslant 0, \\
m_{-s}=m_{s}^{*}, \quad j_{k}=-\mathrm{i} g_{k} / \chi_{0}^{\frac{1}{2}}, \quad k \geqslant 1, \\
j_{-k}=j_{k}^{*}, \quad j_{0}=\mathrm{i}\left(\chi_{0}^{\frac{1}{2}}-\chi_{0}^{* \frac{1}{2}}\right),
\end{array}\right\}
$$

and

i.e.

$$
g_{k}(a, b)=f_{k}(b, a)
$$

$$
\left(1-\xi_{a} t\right)^{\frac{1}{2}}\left(1-\xi_{b} t\right)^{-\frac{1}{2}}=g_{0}+g_{1} t+\ldots
$$

In computing the solutions (find $a, b, \tau_{\mathrm{b}} / u_{\mathrm{b}}$, for given $p_{c} / u_{\mathrm{b}}$ ), it is convenient to choose $a$ as given, and find the corresponding $b, \tau_{\mathrm{b}} / u_{\mathrm{b}}$ and $p_{c} / u_{\mathrm{b}}$. For given $a, b, p_{c} / u_{\mathrm{b}}$ is determined explicitly by (3.18), since $l_{0}$ must be real. The system (3.29) is then solved iteratively to locate $b$, and then $\tau_{\mathrm{b}}$ is computed from (3.30). Evidently, the sliding law can be written in the form

$$
\tau_{\mathrm{b}}=p_{c} f\left(u_{\mathrm{b}} / p_{c}\right) \text {. }
$$

Before proceeding to the results, there are two points to be made. Firstly, although we only prescribe $H^{\prime}(a)=0$, we might expect $H^{\prime}(b)=0$ also, in view of the resultant potentially incompatible singularity in $p+p_{c}$ there (viscous flow at a corner requires a stress singularity). However, we are seemingly not at liberty to prescribe this latter condition. In fact we have found numerically that $H^{\prime}(b)=0$, within numerical error, in all cases. This happy circumstance suggests that one should be able to prove the result, and this is done in Appendix 1, where it is shown that the solution $G$ of this section is such that

$$
H^{\prime}(b)=H^{\prime}(a) \text {. }
$$

This unsuspected result is then a very useful check that the numerical solution is doing its job.

However, one can go further. As already mentioned, it is possible to write a solution to the Hilbert problem using the Plemelj function

$$
\chi_{2}=\left(\zeta-\xi_{a}\right)^{\frac{1}{2}}\left(\zeta-\xi_{b}\right)^{\frac{1}{2}}
$$

as was indeed done in the first draft of this paper, and in the author's doctoral thesis. Then one finds that the condition on $G$ at $\infty$ determines both $a$ and $b$, so that the determination of the cavity thickness $H$ uncouples from that of the Hilbert problem. In general, one would then expect (on solving the boundary value problem for $H$ ) to have $H^{\prime}(a), H^{\prime}(b) \neq 0$. However, it is not difficult to show (using the method of Appendix 1) that (3.34) remains valid in that case also, so that a physical solution with $H \geqslant 0$ does not exist, unless $H^{\prime}(a)=H^{\prime}(b)=0$. There do not seem to be any grounds for supposing that these latter conditions hold, and so we are forced to the conclusion that no physical solution corresponding to (3.35) exists.

One might pursue similar lines of inquiry with the fundamental solution

$$
\chi=\left(\zeta-\xi_{a}\right)^{-\frac{1}{2}}\left(\zeta-\xi_{b}\right)^{-\frac{1}{2}}
$$

but this seems even less physically plausible, and we have not done so. 


\section{RESULTS}

We have solved the problem stated in $\S 3$ numerically, for a variety of bedrocks. We are here restricted to periodic beds with one cavity per period, and this basically means one hump per period. For such beds, the primary result is that the stress exhibits a single maximum at a finite velocity and decreases thereafter. An example is shown in figure 3 , for a sinusoidal bedrock. In figure 4 we show a sequence of cavity shapes for the sinusoidal bedrock. Several features may be remarked on.

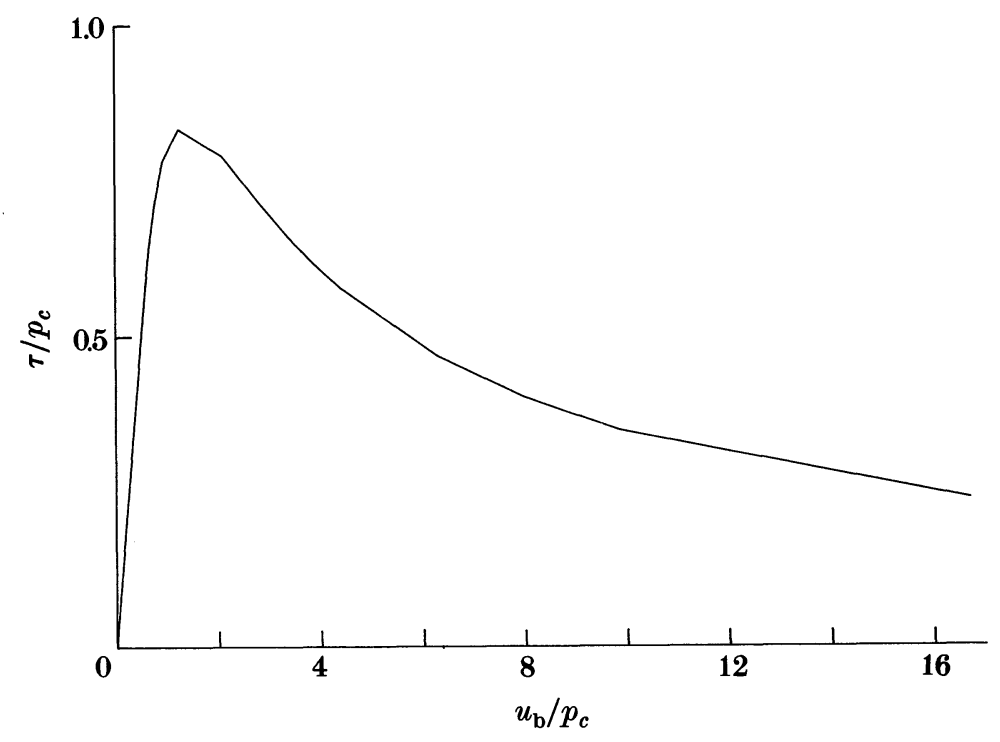

Figure 3. Sliding law for a sinusoidal bed, $h=\cos x($ bed 1$)$.

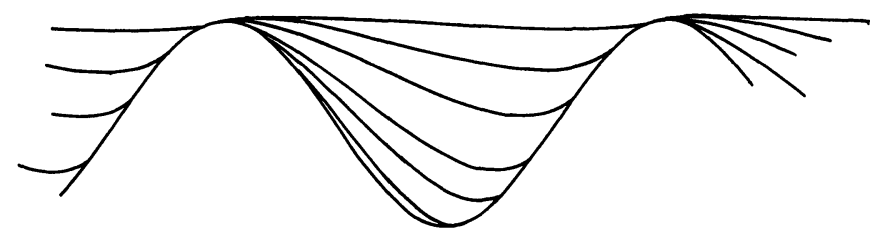

Figure 4. Various cavity shapes for $h=\cos x$, at increasing values of $u_{\mathrm{b}} / p_{c}$.

(i) The cavity roofs are curved. Lliboutry's ( 1968,1979$)$ derivation of the sliding law for a sine wave profile involved the assumption that the cavities became reattached to the bedrock at the intersection with the bed of the tangent at the point of separation. It can be seen from figure 4 that this arbitrary assumption is seemingly well-justified for a sine wave.

(ii) The cavity reattaches to the bed tangentially. This can be seen in figure 4 and later plots, and is proved in Appendix 1. Consequently the ice pressure is continuous at the cavity end points, and no stress discontinuity occurs (though 
the stress field is not analytic). We emphasize that this result is a deduction from the analysis, and is not prescribed $a$ priori, although it is physically sensible.

(iii) Examination of the numerical results for figure 4 reveals that, at high basal velocity $u_{\mathrm{b}}$, the point of separation retreats behind the point where $h$ is a maximum, i.e. it occurs slightly upstream of the bump. In fact, the separation point at first decreases as $u_{\mathrm{b}}$ increases, reaches a minimum, and then increases towards its asymptotic value of zero as $u_{\mathrm{b}}$ tends to infinity. On the other hand, the reattachment point $b$ increases monotonically with $u_{\mathrm{b}}$ towards its asymptote of $2 \pi$. This behaviour is illustrated in figure 5 .

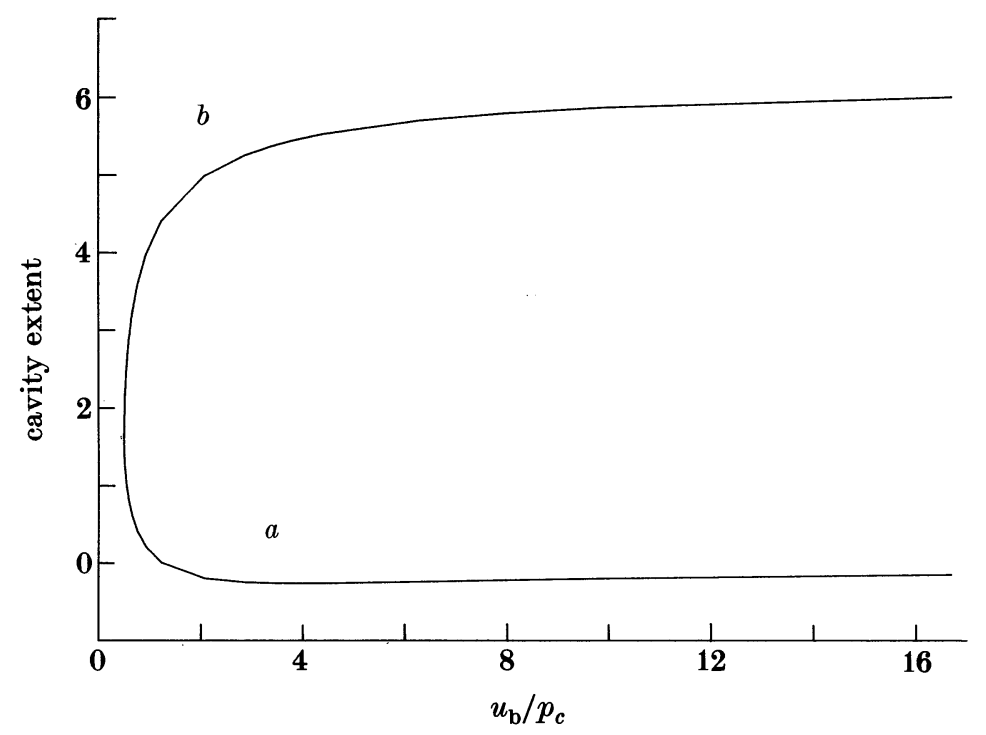

Figure 5. Cavity extent ( $a, b$ values) plotted against $u_{\mathrm{b}} / p_{c}$ for bed 1 .

Despite these nuances, Lliboutry's more heuristic results are qualitatively reproduced. Figure 3 here is essentially identical to Lliboutry's (1979) figure 7, with $n=1$ (Newtonian), so that $T \propto N / u_{\mathrm{b}}$. However, the differences become more pronounced when one looks at non-sinusoidal profiles.

For symmetrical, but isolated bumps, the sliding law shows the same single maximum of stress versus velocity, but a new feature emerges, which is quite unexpected. Since there is no guarantee in advance that only one period per cavity exists, it is checked in the numerical solution that $p+p_{c}>0$ in the uncavitated bedrock $C^{\prime}$. This condition is satisfied for all values of $u_{\mathrm{b}}$ in figure 3 , but not in figure 6 , where the region of invalidity is marked. The one-cavity solution indicates that part of $C^{\prime}$ has $p+p_{c}<0$, and the inevitable conclusion is that a secondary cavity must form where this happens. In figure 7 we show two cavity profiles, for one of which secondary cavitation is indicated by the tinted areas, where $p+p_{c}<0$. The corresponding variation of $p+p_{c}$ with $x$ in the attached region $\left(C^{\prime}\right)$ is shown in figure 8 . One can see that the negative pressure is relatively small, and we do not expect the correct solution to be significantly different. In particular, the shape of the sliding law in figure 6 is correct. 


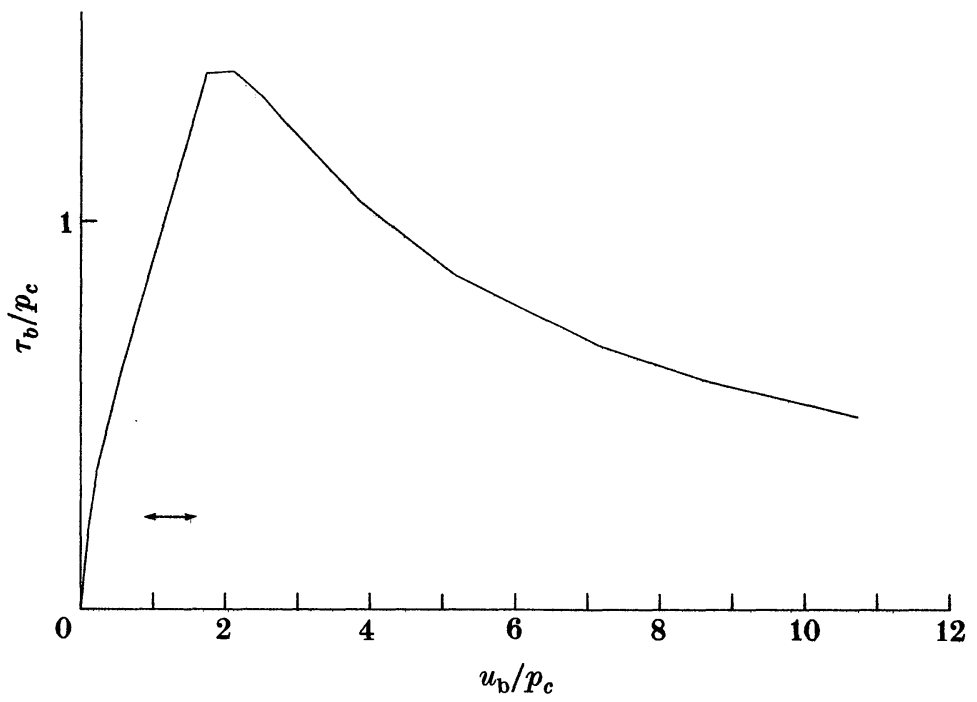

Fraure 6. Sliding law for bed $2, h=\mathrm{e}^{-3 x^{2}}$ in $(-\pi, \pi)$, extended periodically. Arrows indicate the extent of secondary cavitation.

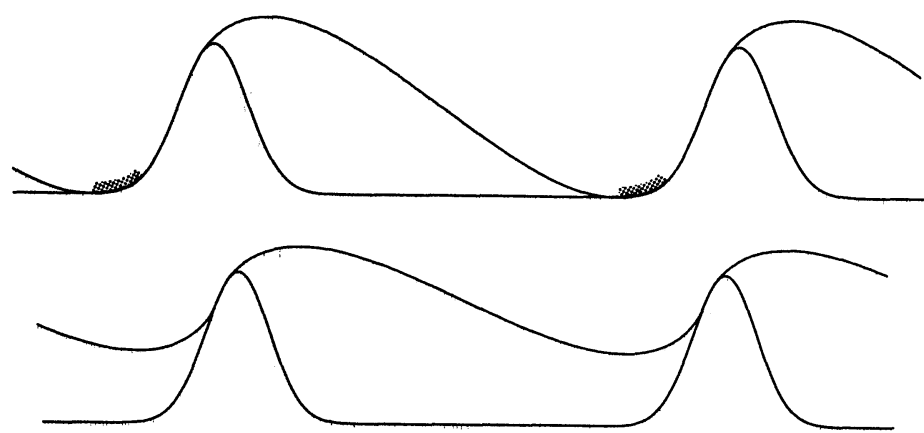

Frgure 7. Two examples of cavity shapes for bed 2 , at $u_{\mathrm{b}} / p_{c}=0.54$ (upper), 0.93 (lower). The upper example shows a zone of secondary cavitation in the tinted region. The corresponding variation of $\left(p+p_{c}\right) / u_{\mathrm{b}}$ with $x$ is shown in figure 8 .

The mechanism of secondary cavitation formation can be understood as follows. If the Fourier components of the bed $h$ are $a_{k}$, then the drag when cavities are absent (equation (2.38)) is proportional to $\sum k^{3}\left|a_{k}\right|^{2}$. Now if $h$ has $(m-1)$ continuous derivatives, but $h^{(m)}$ is discontinuous, then $a_{k} \sim k^{-m}$, as follows on integration by parts. Thus if $h$ is step-shaped, i.e. $h^{\prime}$ is only piecewise continuous, then $\sum k^{3}\left|a_{k}\right|^{2} \sim \sum k$ diverges and consequently cavities form for $a n y u_{\mathrm{b}}>0$. What is less physically obvious is that this is true in the present model if $m=2$, i.e. if $h^{\prime \prime}$ has discontinuities. Consequently, we would expect cavities to form in or near regions where $h^{\prime \prime}$ is discontinuous and even where $h^{\prime \prime}$ is large. This phenomenon is exemplified in figure 7. For smoother stoss faces, secondary cavities disappear, as indicated in figures 9 to 11. Figure 9 shows the sliding law for a skewed bed, and 


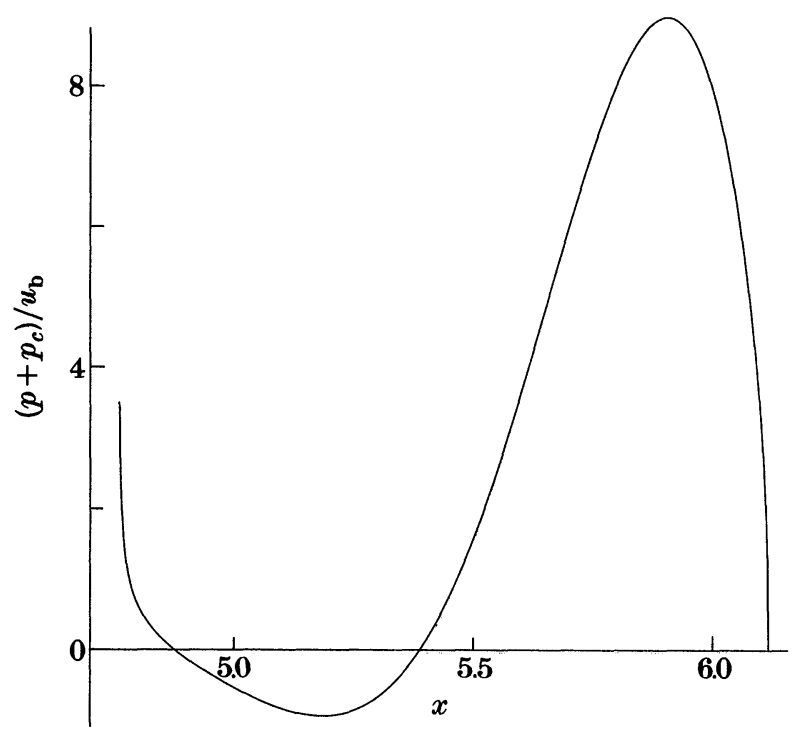

Figure 8. The variation of $\left(p+p_{c}\right) / u_{\mathrm{b}}$ with $x$ in $C^{\prime}$ for the flow corresponding to the upper picture in figure 7. Secondary cavitation is indicated by the negative values around $x=5$.

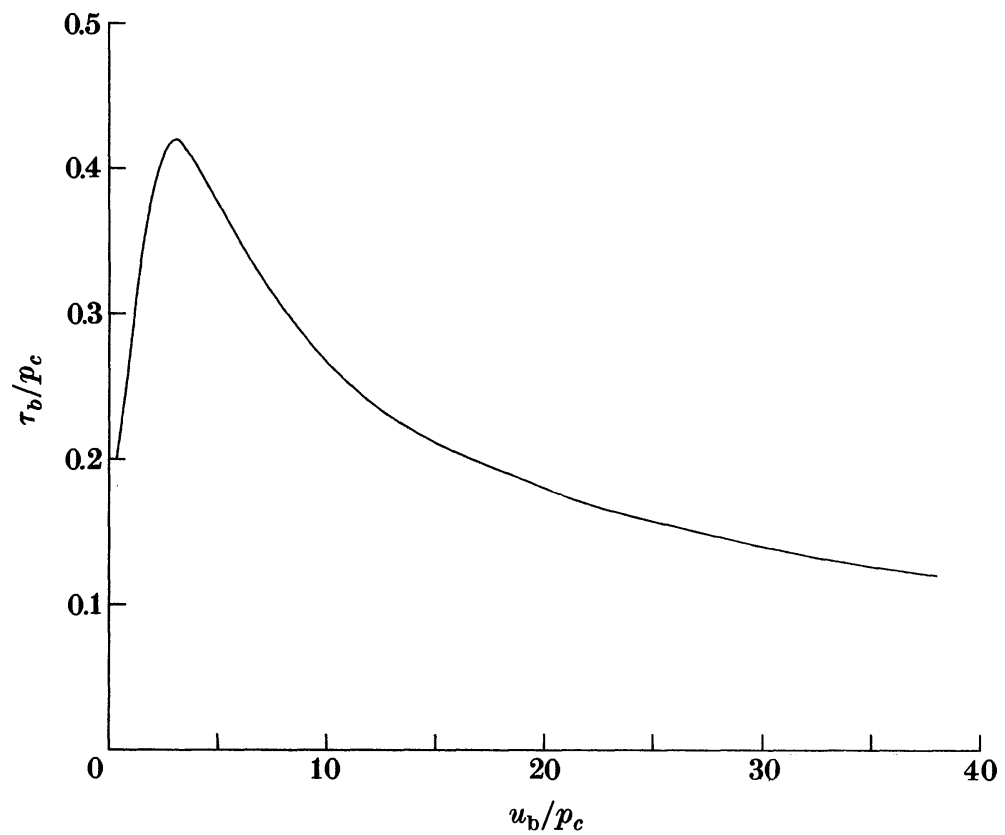

Figure 9. Sliding law (smoothed by Bessel interpolation) for bed $3, h=\mathrm{e}^{-L x^{2}-\epsilon x^{4}}$ in $(-\pi, \pi)$, extended periodically, where $L=[p+q+(p-q) \tanh \{k(x-c)\}] / 2, k=2, c=0.5, p=3$, $q=0.3$ and $\epsilon=0.01$. The term in $\epsilon$, and the elaborate choice of $L$, is so that the bed will appear sufficiently smooth to avoid Gibbs's phenomenon. 

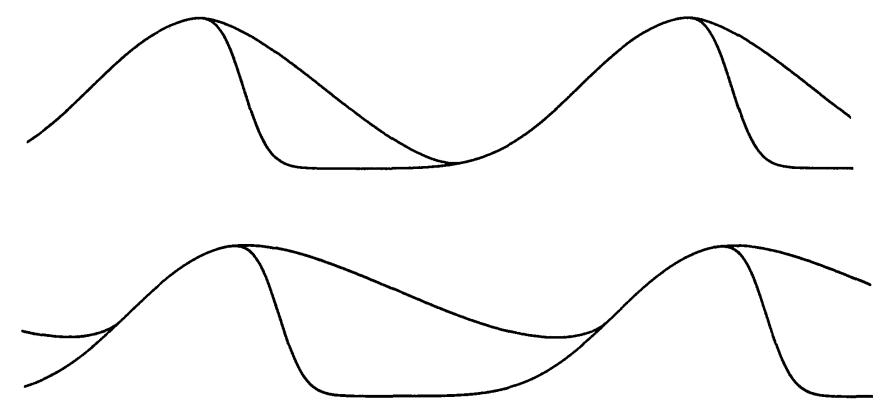

Figure 10. Two examples of cavity shapes for bed 3 , at $u_{\mathrm{b}} / p_{c}=1.49$ (upper) and 3.09 (lower). Notice how straight the cavity roofs are.

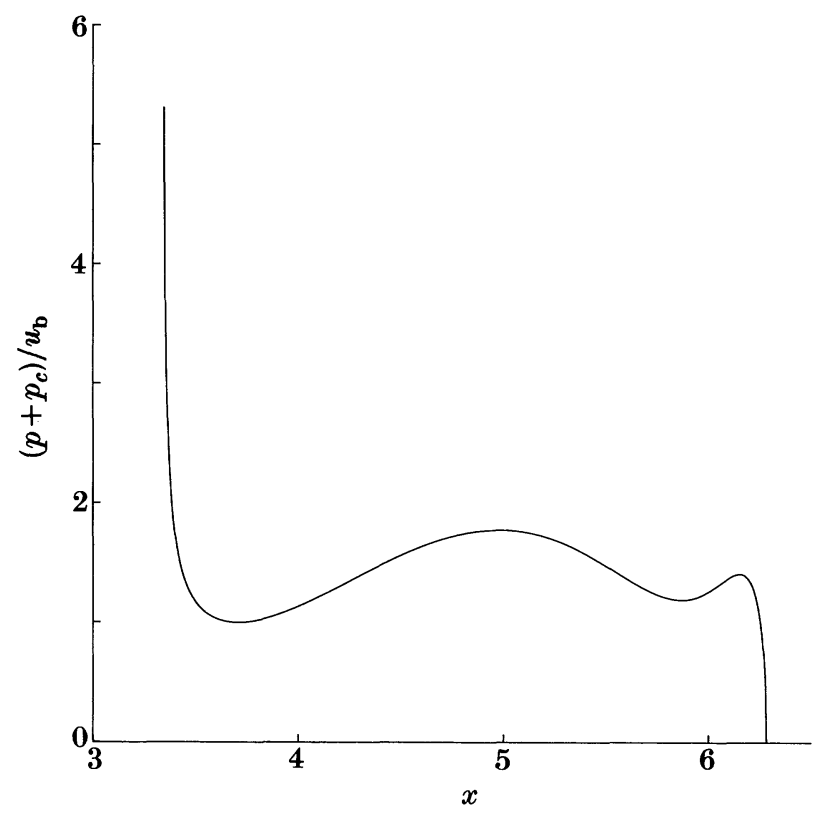

Figure 11. Variation of $\left(p+p_{c}\right) / u_{\mathrm{b}}$ with $x$ in $C^{\prime}$ for the upper picture of figure 10 . There is no secondary cavitation at all for bed 3 .

figures 10 and 11 show two typical cavity shapes and (for one of them) the corresponding variation of $p+p_{c}$ with $x$.

The points (i)-(iii) above are clearer in figure 7. For moderately significant cavitation, the point of separation $a$ is upstream of the bump's maximum, and the tangent there is obviously irrelevant to the position of $b$. However, figure 10 shows the opposite tendency. Another important point which emerges from numerical comparison of figure 6 with figure 7 , is that the stress increases with velocity until the cavity reaches the next bump. In retrospect this is physically obvious, but it highlights Lliboutry's (1979) insistence that periodic (e.g. sinusoidal) bedrocks (and thus sliding laws like figure 3 ) are not practically realistic, since all the bumps are drowned simultaneously. In reality, when bumps of one size are drowned, the 
resistance they offer to the flow will be transferred to larger bumps, and thus stress will in fact always increase with velocity, providing that very large bumps do exist.

\section{Discussion}

We have formulated the Nye-Kamb sliding process (small slope bed, linear flow law) minus regelation, in terms of complex variable theory, and reduced the resulting problem to that of solving a free boundary problem for a second order ordinary differential equation. This is essentially straightforward to do numerically, and results for a variety of bedforms have been given in $\S 4$. The basic result is that, for the single-humped bedrocks studied here, the sliding law is as shown in figure $6 b$ of Hutter ( $1982 a)$, i.e. exhibits a single maximum of stress at a finite velocity, and then the stress decreases towards zero as the velocity tends towards infinity.

This result basically emulates that of Lliboutry ( I 979), and its novelty only lies in that the analysis involves the solution of a precisely stated problem, without ad hoc assumptions. However, Lliboutry also emphasized that real, non-periodic bedrocks will have very different sliding laws, and we can offer some physical explanation for this, to supplement Lliboutry's analytic explanation, which involves a 'shadowing function' for a 'Gaussian' bedrock. The important point is that stress and cavity length increase with velocity until the bumps begin to be drowned. For a random bedrock, what will happen as velocity increases is that, when smaller bumps begin to be drowned, the resistance they offer to the flow will decrease, but the larger bumps will take it up, with a concomitant increase in cavity length. One therefore expects, for a general bedrock, that stress will increase with velocity until the largest bumps become flooded (i.e. the whole bedrock). Only then might the stress decrease but, realistically, this is unlikely to be the case. Then a realistic sliding law would be more like figure $6 d$ of Hutter (1982a), or even figure $6 a$. In particular, these arguments do not suggest a multivalued sliding law ( $u$ a triple-valued function of $\tau$ ).

If this is true, then the dynamical explanation of surges requires an active role of the basal water pressure, and this has recently been proposed on both a theoretical and experimental basis by Kamb et al. (1985). It is hoped in future work to incorporate a 'realistic' sliding law and 'realistic' drainage model into the kinematic wave equation, with the hope of explaining surges deductively.

The sliding law we have deduced is written dimensionlessly in the form

$$
\tau / p_{c}=f\left(u_{\mathrm{b}} / p_{c}\right) .
$$

It is of some interest to write it dimensionally, so that its likely size can be estimated. This is done in Appendix 2. For an applied dimensional stress $\tau_{D}$ dragging ice over a bed $y=[y] h(x /[x])$, so $[x]$ is a horizontal length scale, $[y]$ is the amplitude, then the dimensional velocity $u_{\mathrm{D}}$ far from the bed is given by

$$
\tau_{\mathbf{D}}=N f\left[\frac{\nu \eta u_{\mathrm{D}}}{[x] N} ; h\right]
$$

where

$$
\nu=[y] /[x]
$$


is assumed small, $\eta$ is the (constant) ice viscosity, and $N=p_{I}-p_{\mathrm{w}}$ is the effective pressure. The most obvious ad hoc generalization for a non-Newtonian flow law would be to replace $\eta$ in (5.2) by

$$
\eta=1 / B \tau_{\mathrm{D}}^{n-1}
$$

where $B$ is the constant in Glen's flow law. (5.2) can then be written as

$$
\begin{gathered}
\tau_{\mathrm{D}}=\nu N g\left[\frac{\nu^{2} u_{\mathrm{D}}}{B[x] \tau_{\mathrm{D}}^{n}} ; h\right], \\
\tau_{\mathrm{D}}=\nu N G\left[\frac{\nu^{n-2} B[x] N^{n}}{u_{\mathrm{D}}}\right],
\end{gathered}
$$

which resembles Lliboutry's (1979) relation (108) with, however, an extra factor of $\nu^{2}$ in the argument of $G$. However, the exponent $n-2$ becomes $n-4$, if one puts

$$
\eta=1 / B\left(\tau_{\mathrm{D}} / \nu\right)^{n-1},
$$

which is more appropriate since $\tau_{\mathrm{D}} / \nu$ is the order of the stresses near the bed. Then (5.6) reproduces Lliboutry's result with $n=3$.

Let $P$ be the ice overburden pressure, and $\epsilon$ the surface slope of a glacier or ice sheet. Then

$$
N / P \lesssim 1, \quad \tau_{\mathrm{D}} \approx \epsilon P .
$$

We compare $u_{\mathrm{D}}$ with the velocity due to shearing of the ice, $u_{\mathrm{sh}}$. We can expect

$$
\tau_{\mathrm{D}} \sim \eta u_{\mathrm{sh}} / d
$$

where $d$ is the ice thickness; thus (5.2) is

$$
\frac{\epsilon}{\nu} \approx \frac{N}{P} f\left[\left\{\frac{\nu \epsilon d}{[x](N / P)}\right\} \frac{u_{\mathrm{D}}}{u_{\mathrm{sh}}}\right] .
$$

With $\nu \sim 0.1-0.2$, we expect $\epsilon / \nu \sim 1$ for glaciers $(\epsilon \sim 0.1)$ but $\epsilon / \nu \ll 1$ for ice sheets. Correspondingly

$$
\frac{u_{\mathrm{D}}}{u_{\mathrm{sh}}} \sim \frac{[x]}{\nu \epsilon d}
$$

for glaciers, whereas cavitation is likely to be irrelevant to large ice sheets. One sees from (5.11) that values $[x]=1 \mathrm{~m}, d=100 \mathrm{~m}, \epsilon=\nu=0.1$ lead to sliding speeds comparable to those due to shearing within the ice, and can be larger or smaller depending on the roughness of the bed. If, however, water pressure is high $(N \ll P),(5.10)$ requires $u_{\mathrm{D}}$ to be large. For example, if $[x] \sim \nu \epsilon d$, and if $f(\beta) \sim \beta^{\gamma}$ as $\beta \rightarrow \infty$ with $0<\gamma<1$, then (5.10) implies

$$
u_{\mathrm{D}} / u_{\mathrm{sh}} \sim(N / P)^{-(1-\gamma) / \gamma} \quad \text { as } \quad N / P \rightarrow 0,
$$

so that basal sliding increases algebraically as $N \rightarrow 0$. There exist some experimental (Budd et al. 1979) and field (Bindschadler 1983) measurements which seem to lend support to a 'generalized Weertman' law of the form $f(\beta) \propto \beta^{\gamma}$, or generally

$$
\tau \propto u^{a} N^{b},
$$

where these authors found $a, b \approx \frac{1}{3}$. It should be pointed out, however, that Budd's 
laboratory experiments are not obviously applicable to large scale glacier sliding, and that Bindschadler's analysis of his field data involves several questionable assumptions. More effort in this direction is required. Equation (5.13) is also consistent with field results of Iken (personal communication) who finds $u$ becoming large as $N$ approaches ('approximately') zero.

The results given here may be used as stepping stones towards the determination of such sliding laws for more realistic bedforms.

I am grateful to Alan Jones, who perceived at Interlaken in 1983 that I had got the singularity at the cavity end point wrong; also to Adam Wheeler, for much advice and assistance in using the VAX 11/780 at Oxford University, on which the computations were done.

\section{APPENDIX 1}

In this appendix, we show that the cavity thickness $H(x)$ satisfies the relation

$$
H^{\prime}(a)=H^{\prime}(b)
$$

The solution of (3.2) can be written in the form

$$
G(\zeta)=\frac{\chi(\zeta)}{2 \pi \mathrm{i}} \int_{C^{\prime}} \frac{f(\tau) \mathrm{d} \tau}{\chi^{+}(\tau)(\tau-\zeta)}+G_{\infty} \chi
$$

where

$$
\chi=\left(\frac{\zeta-\xi_{a}}{\zeta-\xi_{b}}\right)^{\frac{1}{2}}
$$

with $\chi \sim 1$ as $\zeta \rightarrow \infty$. Here

$$
f(\tau)=u_{\mathrm{b}} h_{2}(\tau), \quad G_{\infty}=-\mathrm{i} p_{c} / 4,
$$

so that

$$
f(\tau)=\sum_{-\infty}^{\infty} c_{n} \tau^{n}, \quad c_{0}=0
$$

Since we require $G(0)=-G_{\infty}$, (A 1.2) implies

$$
\frac{\chi_{0}}{2 \pi \mathrm{i}} \int_{C^{\prime}} \frac{f(\tau) \mathrm{d} \tau}{\tau \chi^{+}(\tau)}+G_{\infty} \chi_{0}=-G_{\infty}
$$

where $\chi(0)=\chi_{0}$.

From (3.3) and (A 1.4), the condition $\left[H^{\prime}\right]_{a}^{b}=0$ is equivalent to

$$
I=\int_{C}\left[G-\frac{1}{2} f(\tau)\right] \frac{\mathrm{d} \tau}{\tau}=0,
$$

remembering that $\mathrm{d} \xi=\mathrm{i} \xi \mathrm{d} x$. We undertake to prove (A 1.7). By completing the contour round the inside of $C^{\prime}$ (see figure 1), we have

$$
\left.\begin{array}{rl}
I & =\oint_{C \cup C^{\prime}}\left[G^{+}-\frac{1}{2} f(\tau)\right] \frac{\mathrm{d} \tau}{\tau}-\int_{C^{\prime}}\left[G^{+}-\frac{1}{2} f(\tau)\right] \frac{\mathrm{d} \tau}{\tau}, \\
& =-2 \pi \mathrm{i} G_{\infty}-\int_{C^{\prime}}\left[G^{+}-\frac{1}{2} f(\tau)\right] \frac{\mathrm{d} \tau}{\tau},
\end{array}\right\}
$$


using residue calculus on the first integral, and that $c_{0}=0$ (A 1.5). From (A 1.2) and the Plemelj formulae,

and so

$$
G^{+}=\chi^{+}\left[\frac{1}{2 \pi \mathrm{i}} f_{C^{\prime}} \frac{f(\tau) \mathrm{d} \tau}{\chi^{+}(\tau)(\tau-\xi)}+\frac{f(\xi)}{2 \chi^{+}(\xi)}\right]+G_{\infty} \chi^{+}
$$

$$
\left.\begin{array}{rl}
J & =\int_{C^{\prime}}\left[G^{+}-\frac{1}{2} f(\tau)\right] \frac{\mathrm{d} \tau}{\tau} \\
& =\int_{C^{\prime}} \frac{\chi^{+}(\xi)}{\xi} \mathrm{d} \xi\left[G_{\infty}+\frac{1}{2 \pi \mathrm{i}} \oint_{C^{\prime}} \frac{f(\tau) \mathrm{d} \tau}{\chi^{+}(\tau)(\tau-\xi)}\right] .
\end{array}\right\}
$$

From (A 1.8) we wish to show

$$
J=-2 \pi \mathrm{i} G_{\infty} .
$$

We evaluate $J$ in two parts. Firstly, the function $g=\chi$ satisfies $g^{+}-g^{-}=2 \chi^{+}$, and so by the discontinuity theorem, we can write

$$
\chi=\frac{1}{2 \pi \mathrm{i}} \int_{C^{\prime}} \frac{2 \chi^{+}(\tau) \mathrm{d} \tau}{\tau-\zeta}+1
$$

since $\chi \sim 1$ as $\zeta \rightarrow \infty$. Therefore

$$
J_{1}=\int_{C^{\prime}} \frac{\chi^{+}(\xi) \mathrm{d} \xi}{\xi}=\mathrm{i} \pi\left(\chi_{0}-1\right)
$$

Secondly, we can change the order of integration in the repeated integral, by the Poincaré-Bertrand formula (since only one integral is singular). Then

$$
\begin{aligned}
J_{2} & =\frac{1}{2 \pi \mathrm{i}} \int_{C^{\prime}} \chi^{+}(\xi) \frac{\mathrm{d} \xi}{\xi} f_{C^{\prime}} \frac{f(\tau) \mathrm{d} \tau}{\chi^{+}(\tau)(\tau-\xi)}, \\
& =\frac{1}{2 \pi \mathrm{i}} \int_{C^{\prime}} \frac{f(\tau) \mathrm{d} \tau}{\tau \chi^{+}(\tau)} f_{C^{\prime}} x^{+}(\xi) \mathrm{d} \xi\left[\frac{1}{\xi}+\frac{1}{\tau-\xi}\right], \\
& =\frac{1}{2 \pi \mathrm{i}} \int_{C^{\prime}} x^{+}(\xi) \frac{\mathrm{d} \xi}{\xi} \int_{C^{\prime}} \frac{f(\tau) \mathrm{d} \tau}{\tau \chi^{+}(\tau)}-\frac{1}{2 \pi \mathrm{i}} \int_{C^{\prime}} \frac{f(\tau) \mathrm{d} \tau}{\tau \chi^{+}(\tau)} f_{C^{\prime}} \frac{\chi^{+}(\xi) \mathrm{d} \xi}{\xi-\tau} .
\end{aligned}
$$

The Plemelj formulae applied to (A 1.12) imply that on $C^{\prime}$

so that

$$
\chi^{+}(\xi)=1+\frac{1}{2 \pi \mathrm{i}} \oint_{C^{\prime}} \frac{2 \chi^{+}(\tau) \mathrm{d} \tau}{\tau-\xi}+\chi^{+}(\xi)
$$

$$
\frac{1}{2 \pi \mathrm{i}} \oint_{C^{\prime}} \frac{\chi^{+}(\xi) \mathrm{d} \xi}{\xi-\tau}=-\frac{1}{2}
$$

Substituting into the last integral in (A 1.14), we find

i.e. $\quad J_{2}=\left(\frac{1}{2}+\frac{J_{1}}{2 \pi \mathrm{i}}\right) \int_{C^{\prime}} \frac{f(\tau) \mathrm{d} \tau}{\tau \chi^{+}(\tau)}$.

$$
J_{2}=\frac{1}{2 \pi \mathrm{i}} \int_{C^{\prime}} \frac{\chi^{+}(\xi)}{\xi} \mathrm{d} \xi \int_{C^{\prime}} \frac{f(\tau) \mathrm{d} \tau}{\tau \chi^{+}(\tau)}+\frac{1}{2} \int_{C^{\prime}} \frac{f(\tau) \mathrm{d} \tau}{\tau \chi^{+}(\tau)}
$$


Now the integral in (A 1.16) is given by (A 1.6). Since $J=G_{\infty} J_{1}+J_{2}$, we simply substitute (A 1.6) into (A 1.16), and thence into (A 1.10) using (A 1.13), and the result (A 1.11), and (A 1.7) and (A 1.1) follow.

The calculation goes through exactly the same way for $\chi=\chi_{2}$ given by (3.35).

\section{APPENDIX 2}

Here we retrieve the dimensional sliding law from the dimensionless form (5.1):

$$
\tau / p_{c}=f\left(u_{\mathrm{b}} / p_{c}\right) \text {. }
$$

In terms of the original dimensional units (denoted by a suffix $D$ ) in $\S 2$, we have

$$
\tau=\tau_{\mathrm{D}} /[\tau], \quad u_{\mathrm{b}}=u_{\mathrm{D}} / U, \quad p_{c}=\nu N /[\tau],
$$

where $N$ is the effective pressure, $=p_{I}-p_{w}$. Thus (A 2.1) is

$$
\frac{\tau_{\mathrm{D}}}{\nu N}=f\left[\frac{u_{\mathrm{D}}[\tau]}{U \nu N}\right] .
$$

From (2.5), $[\tau]=\eta U / d$, where $\eta$ is viscosity and $d$ would be a typical global depth scale. However, the local problem can be posed apparently without reference to a large length scale. In this case, one simply defines $d$ via (2.2) and (2.13). It is necessary to do this in order that (2.14)-(2.16) be the correctly stated version of the problem (e.g. so that stress is absent to leading order at infinity). Then using (2.5) and (2.13), we find that (A 2.3) gives

as given in the text at (5.2).

$$
\frac{\tau_{\mathrm{D}}}{\nu N}=f\left[\frac{u_{\mathrm{D}} \eta \nu}{N[x]}\right],
$$

\section{REFERENCES}

Bindschadler, R. 1983 The importance of pressurized subglacial water in separation and sliding at the glacier bed. J. Glaciol. 29, 3-19.

Budd, W. F., Keage, P. L. \& Blundy, N. A. 1979 Empirical studies of ice sliding. J. Glaciol. 23, $157-180$.

England, A. H. I97 I Complex variable methods in elasticity. (181 pages.) London: Wiley-Interscience.

Fowler, A. C. 198I A theoretical treatment of the sliding of glaciers in the absence of cavitation. Phil. Trans. R. Soc. Lond. A 298, 637-685.

Fowler, A. C. 1982 Waves on glaciers. J. Fluid Mech. 120, 283-321.

Fowler, A. C. 1984 On the transport of moisture in polythermal glaciers. Geophys. Astrophys. Fluid Dynam. 28, 99-140.

Hodge, S. M. 1974 Variations in the sliding of a temperate glacier. J. Glaciol. 13, 349-369.

Hutter, K. I982a Dynamics of glaciers and large ice masses. A. Rev. Fluid Mech. 14, 87-130.

Hutter, K. I982b A mathematical model of polythermal glaciers and ice sheets. Geophys. Astrophys. Fluid Dynam. 21, 201-224.

Iken, A. 198I The effect of the subglacial water pressure on the sliding velocity of a glacier in an idealized numerical model. J. Glaciol. 27, 407-421.

Kamb, W. B. 1970 Sliding motion of glaciers: theory and observation. Rev. Geophys. Space Phys. 8, 673-728.

Kamb, W. B., Raymond, C. F., Harrison, W. D., Engelhardt, H., Echelmeyer, K. A., Humphrey, N., Brugman, M. M. \& Pfeffer, T. 1985 Glacier surge mechanism: 1982-1983 Surge of Variegated Glacier, Alaska. Science, Wash. 227, 469-479. 
Lliboutry, L. A. 1968 General theory of subglacial cavitation and sliding of temperate glaciers. J. Glaciol. 7, 21-58.

Lliboutry, L. A. 1975 Loi de glissement d'un glacier sans cavitation. Annls Géophys. 31, 207-225.

Lliboutry, L. A. 1976 Physical processes in temperate glaciers. J. Glaciol. 16, 151-158.

Lliboutry, L. A. 1978 Glissement d'un glacier sur un plan parsemé d'obstacles hémisphériques. Annls Géophys. 34, 147-162.

Lliboutry, L. A. 1979 Local friction laws for glaciers: a critical review and new openings. J. Glaciol. 23, 67-95.

Morland, L. W. I976a Glacier sliding down an inclined wavy bed. J. Glaciol. 17, 447-462.

Morland, L. W. $1976 b$ Glacier sliding down an inclined wavy bed with friction. J. Glaciol. 17, 463-477.

Nye, J. F. I 969 A calculation on the sliding of ice over a wavy surface using a Newtonian viscous approximation. Proc. R. Soc. Lond. A 311, 445-477.

Nye, J. F. I 970 Glacier sliding without cavitation in a linear viscous approximation. Proc. $R$. Soc. Lond. A 315, 381-403.

Nye, J. F. 1973 The motion of ice past obstacles. In: Physics and chemistry of ice (ed. E. Whalley, S. J. Jones \& L. W. Gold), pp. 387-394. Royal Society of Canada, Ottawa.

Oakberg, R. G. 198I Variational methods for glacier mechanics problems. J. Glaciol. 27, 19-24.

Richardson, S. 1973 On the no-slip boundary condition. J. Fluid Mech. 59, 707-719.

Robin, G. de Q. 1976 Is the basal ice of a temperate glacier at the pressure melting point? J. Glaciol. 16, 183-196.

Röthlisberger, H. 1972 Water pressure in intra- and subglacial channels. J. Glaciol. 11, 177-203.

Weertman, J. 1957 On the sliding of glaciers. J. Glaciol. 3, 33-38.

Weertman; J. 1964 The theory of glacier sliding. J. Glaciol. 5, 287-303.

Weertman, J. 197I In defense of a simple model of glacier sliding. J. geophys. Res. 76, $6485-6487$.

Weertman, J. 1972 General theory of water flow at the base of a glacier or ice sheet. Rev. Geophys. Space Phys. 10, 287-333.

Weertman, J. 1979 The unsolved general glacier sliding problem. J. Glaciol. 23, 97-115. 